\title{
SUREICAL MASK USE IN PHYSICAL EXERCISE IN YOUNG HEALTHY SUBJECTS SUME TRIAL
}

\author{
Hugo Mendonça CaféA, B, D
}

Hospital Particular do Algarve, Gambelas | Medicine and Biomedical Sciences Faculty, University of Algarve, Portugal ORCID: 0000-0001-8267-3847 | e-mail: hfcafe@ualg.pt

\author{
Marta Leitão $0^{B}$ \\ Hospital Particular do Algarve, Gambelas, Portugal \\ ORCID: 0000-0002-9006-941
}

\author{
Anya Freitas ${ }^{A, B}$ \\ Faro Health Center, Portugal \\ ORCID: 0000-0002-2162-8548
}

\author{
Ana Marreiros ${ }^{\complement}$ \\ Medicine and Biomedical Sciences Faculty, University of Algarve, Portugal \\ ORCID: 0000-0001-9410-4772

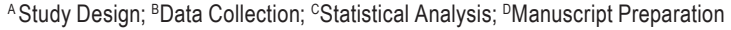

\begin{abstract}
Ahstract In the context of the COVID-19 pandemic, the use of surgical masks has become the new normal. The use of these devices in exercise and medical situations has been advocated with the purpose of reducing contagions, but some concerns exist regarding its impact of physical fitness and safety of use. If the use of mask while exercising can cause decreased functional capacity or dangerous hypoxemia is still something we know little of. Therefore, we performed maximal treadmill stress tests in 12 healthy young subjects, with and without surgical mask use, and measured exercise capacity, oxygen saturation (rest, peak exercise and post-exercise) and electrocardiographic changes on a standard treadmill test. Exercise capacity and oxygen saturation levels decreased in peak exercise vs rest in a statistically significant manner when mask was used. ECG changes, although not significant, were present in 3 subjects when mask was used and disappeared when the test was made unmasked. We concluded that masked exercise has the potential to cause decreased exercise load and oxygen saturation and potentially cause diagnostic errors in medical exams.
\end{abstract}

Key WOrdls surgical mask, exercise, treadmill test, stress test, oxygen saturation, Covid-19, EC 


\section{Introduction}

In the present SARS-COV-2 pandemic, one of the measures that has been progressively implemented by the various nations is related to the mandatory use of a mask in order to reduce the spread of the pathology (CDC, 2020; $E C D C, 20202)$. The locations in which the use of a mask is mandatory vary, with some nations having mandatory use in any public space and others only in non-ventilated places (Greenhalgh, 2020).

An issue that proves to be important has to do with physical exercise. Given that exercise has the potential to generate high levels of aerosols, it has been advocated that a use of a mask should be kept during all activity in order to potentially reduce the risk of viral transmission (Chandrasekaran, Fernandes, 2020). The effect of such mask in physical activity, however, is not clear. Concerns exist on its effect on exercise capacity (decreasing exercise load) and possibility of causing significant hypoxemia. A study by Fikenzer et al. (2020) showed significant negative changes in ventilation parameters in an cycle ergometer test in healthy subjects wearing surgical and FFP2 masks. During our study, a paper published by Shaw et al (Shaw, Butcher, Ko, Zello, Chilibeck, 2020) showed that time to exhaustion during a cycle ergometer test was not different with or without surgical mask use, neither was arterial oxygenation.

On the other hand, in the context of the COVID-19 pandemic, specific exams in cardiology such as the electrocardiogram (ECG), stress test (treadmill or cycle ergometer), are sometimes done with masked patients in order to avoid possible viral spread, given that this test produces significant amounts of aerosols. We do not know, however, whether the use of a mask causes significant changes in cardiovascular parameters in order to interfere in the interpretation of the exam.

We, therefore, aimed to evaluate the impact of the use of a standard surgical mask in a treadmill stress test in young healthy volunteers, in terms of ECG changes, oxygen saturation and total exercise capacity.

\section{Material and Methods}

The study protocol was presented to the Ethics Commission of Hospital Particular do Algarve, that approved its methodology and objectives and confirmed that the study was in accordance with the Declaration of Helsinki.

12 self reported healthy volunteers (medical students and nurses), 4 male and 8 female, with average characteristics presented in Table 1, were asked to perform a Treadmill Stress Test - Bruce protocol, a widely used protocol for evaluation of physical fitness and cardiovascular risk (Bruce, Blackmon, Jones, Strait, 1963; Fletcher et al., 2013; Garner, Pomeroy, Arnold, 2017) - while wearing a standard surgical mask in which total physical capacity, ECG changes and oxygen saturation were evaluated. The tests were stopped after the subjects considered that they had reached exhaustion, therefore requesting termination of the exam. Subjects were asked for a personal opinion if they believed the use of surgical mask interfered with their performance. Two weeks after the first exam, a second one was done, this time without surgical mask (two days before they were required to have a negative SARS-COV-2 test).

The treadmill test was performed in a BTL-770M device, with blood pressure measurements done with a Heine Gamma XXL LF device. Oxygen saturation was evaluated by pulse oximetry with a Sangool FS20D device. A cardiologist and a cardiology technician were present at all times during the tests.

In terms of ECG changes, they were interpreted by a cardiologist in accordance with ACC guidelines (Gibbons et al., 1997). Arterial saturation was measured directly in one of the fingers of the subject before exercise, at peak 
exercise and post-exercise (5 minutes after the end of the exam), and total physical capacity estimated from the total time of exercise. Since different stages are involved in Bruce Protocol ${ }^{7}$ (The BRUCE protocol uses different elevations of the treadmill and speeds used - associated to a specific Metabolic Equivalent (MET) per stage - for instance, stage 1 of the Bruce protocol is performed at 1.7 miles per hour ( $\mathrm{mph}$ ) and a 10\% inclination gradient, stage 2 is $2.5 \mathrm{mph}$ and $12 \%$, while Stage 3 goes to $3.4 \mathrm{mph}$ and $14 \%$, and so on. Each of these stages has a corresponding MET value, which meant that a subject increasing 10 seconds in total exercise time while finishing the test in stage 3 would do significant more workload than one increasing those seconds in stage 1), we created a MET-Score in which the result was obtained by multiplying the seconds of exercise done by the METs associated with the stage in which the exercise was done, then we made the sum of all stages, therefore corresponding to an estimate of the full load of exercise performed.

All data was delivered to a data manager for post-processing.

All subjects received a document explaining the study and signed a consent form authorizing use of their data and agreeing to participate in the study.

In order to maintain consistency, we provided surgical masks to all patients. The mask was an OP-MASK B, a type IIR medical device.

Table 1. General characteristics of the sample (SD - Standard Deviation, BSA - Body Surface Area)

\begin{tabular}{lcccc}
\hline & Male & Female & Total & p-Value \\
\hline $\mathrm{n}$ & 4 & 8 & 12 & 0.283 \\
Age (mean \pm SD) & $30.5 \pm 5.69$ & $27.3 \pm 3.80$ & $28.3 \pm 4.60$ & 0.214 \\
BMl (mean \pm SD) & $24.9 \pm 3.22$ & $22.0 \pm 1.95$ & $23.0 \pm 2.70$ & $0.004<0.05$ \\
Weight (mean \pm SD) & $78.0 \pm 7.44$ & $57.6 \pm 5.00$ & $64.4 \pm 11.50$ & $\mathbf{0 . 0 0 4}<0.05$ \\
Height (mean \pm SD) & $1.8 \pm 0.10$ & $1.62 \pm 0.02$ & $1.67 \pm 0.09$ & $\mathbf{0 . 0 0 4}<0.05$ \\
BSA (mean \pm SD) & $1.10 \pm 0.12$ & $1.6 \pm 0.07$ & $1.7 \pm 0.19$ & 0.05 \\
\hline
\end{tabular}

\section{Statistical analysis}

We performed a statistical analysis using mean and standard deviation. Hypotheses tests were executed for independent samples - Mann-Whitney $U$ test - and for paired samples - Wilcoxon signed rank test and Chi squared test to test association between categorical variables.

IBM SPSS version 27 software was used for the statistical analysis.

\section{Results}

All but one of the subjects had a higher MET-Score while exercising without a mask. Comparing groups, there was a slight but statistically significant increase in MET-Score when subjects performed without a mask, showing that the subjects were able to increase exercise load when the mask was removed (Figure 1). 


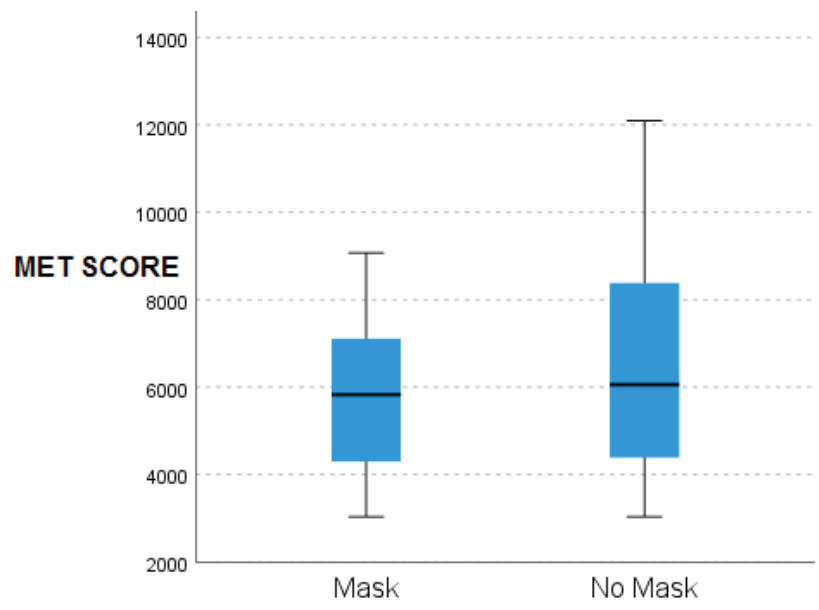

Figure 1. Increased MET-Score mean in No Mask group $(p=0.023)$

Half of the participants declared feeling a sensation of discomfort while wearing the mask. This was also a statistically significant result.

Saturation levels were evaluated in rest, peak exercise and post-exercise (5 minutes after stopping the test). We compared basal and peak levels in one variable ( $\triangle$ SAT1, difference between basal saturation and peak exercise saturation) and peak and post-exercise levels in another ( $\triangle \mathrm{SAT2}$, difference between peak exercise saturation and post-exercise saturation). We found that the decrease of $\Delta S A T 1$ was more apparent in the masked group, and this finding had statistical significance (Figure 2). This was closely related with a statistically significant difference in peak exercise saturation (Figure 3). $\triangle \mathrm{SAT} 2$ was not statistically significant.

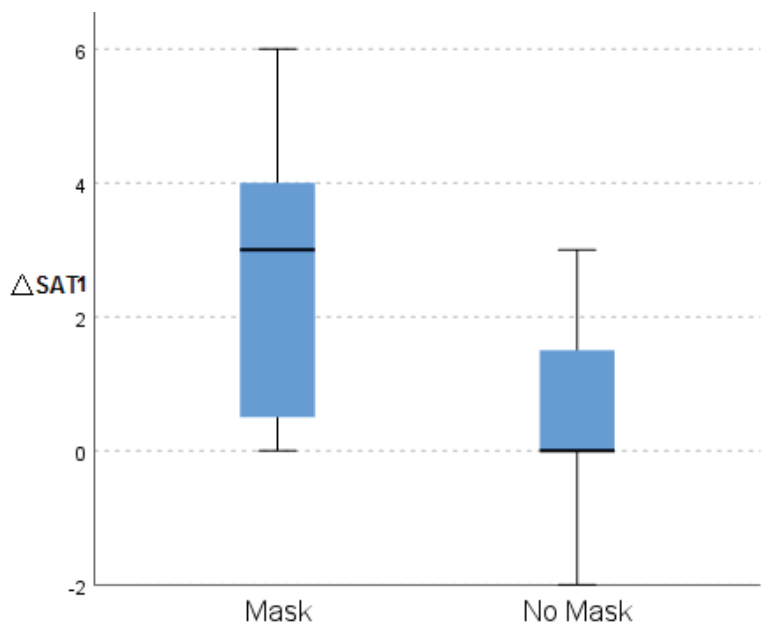

Figure 2. Clear difference between $\Delta$ SAT1 means in both groups (lower the better) $(p=0.021)$ 


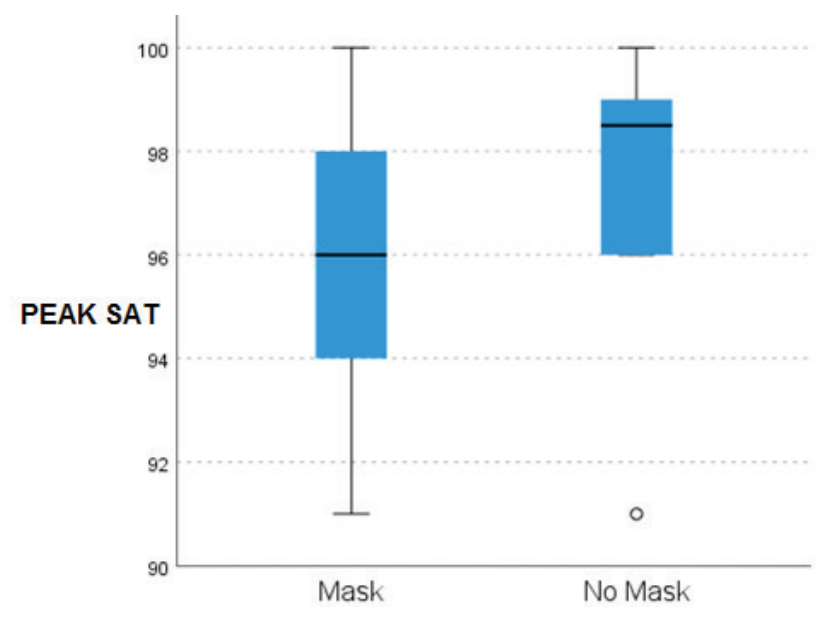

Figule 3. Lower saturations observed in peak exercise when the subjects wore mask (higher the better) $(p=0.049)$

ECG changes were evaluated by a trained cardiologist. In the masked group 3 subjects, all female, had ECG changes that albeit not diagnostic for coronary ischemia, were at least doubtful. Two subjects had slight ST depression (one in inferior leads, the other in V4-V6 leads, neither reaching $1 \mathrm{~mm}$ depression but over $0.5 \mathrm{~mm}$ ) and the other had premature ventricular beats (figures 4, 5 and 6). There changes were not the typical "upslope ST segment" expected in exercise, but a more horizontal change with less than $10 \mathrm{~mm} / \mathrm{second}$ upstroke. Also relevant, none of these changes appeared in peak exercise (two in recovery, another at medium level of effort). When the subjects repeated the test unmasked, these alterations were no longer present.

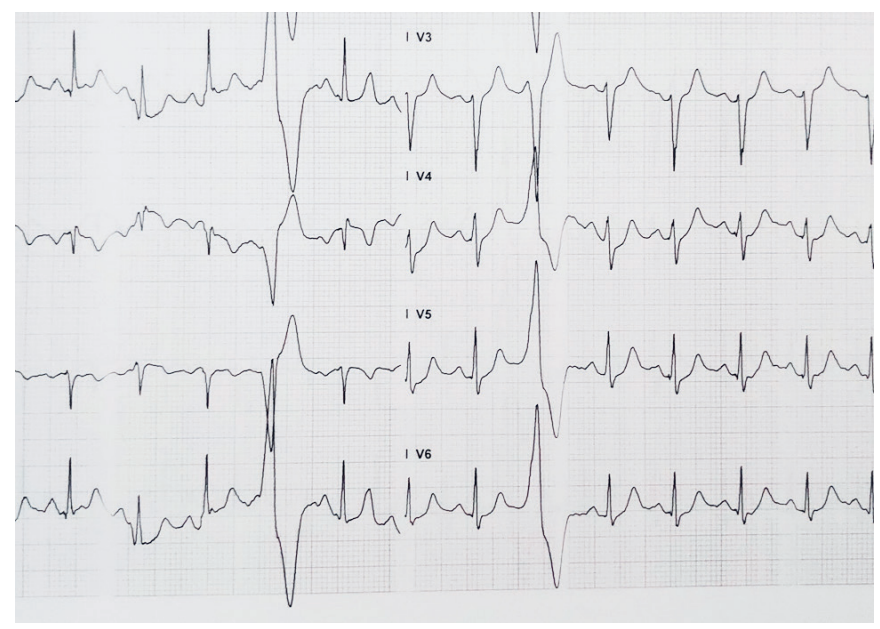

Figure 4. Premature ventricular beats in recovery phase (masked group) 


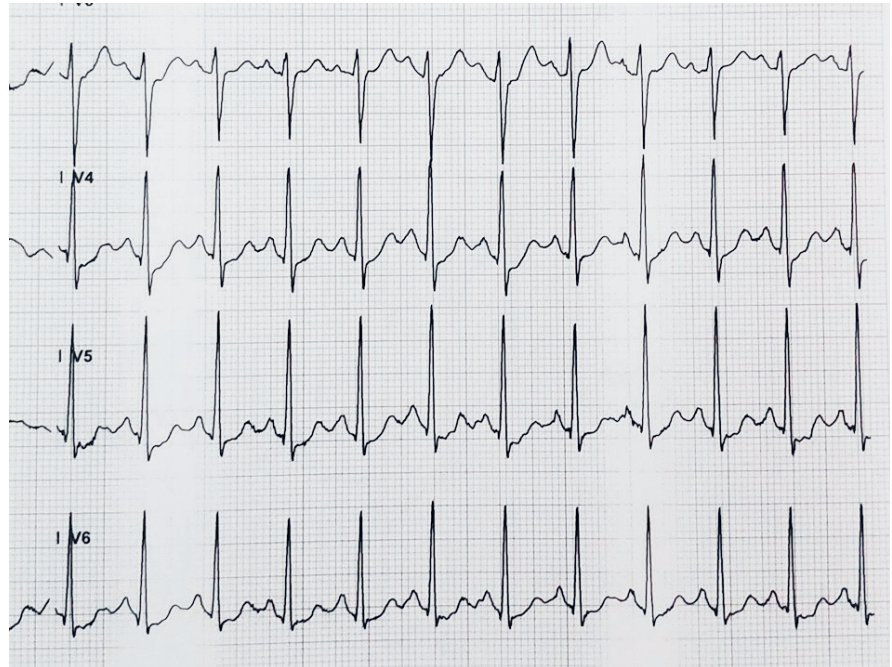

Figure 5. Slight ST depression V4-V6 leads during exercise (masked group)
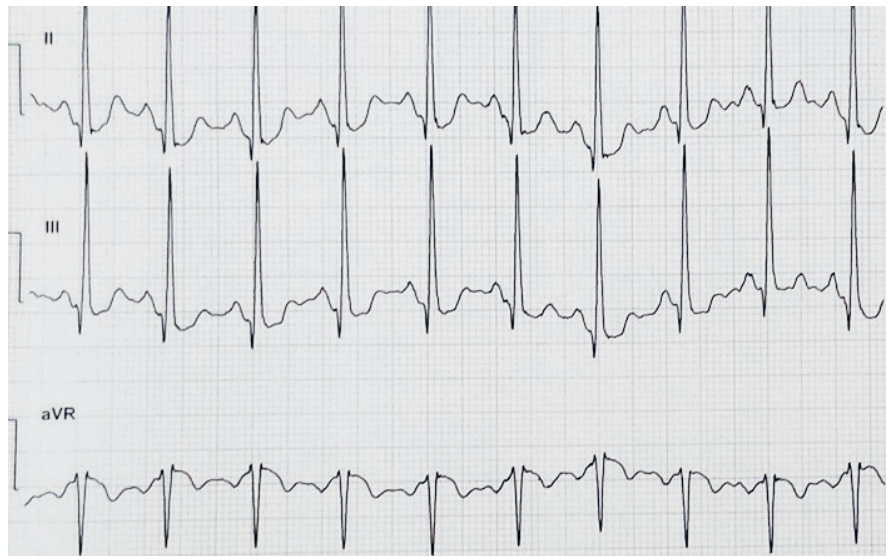

aVL

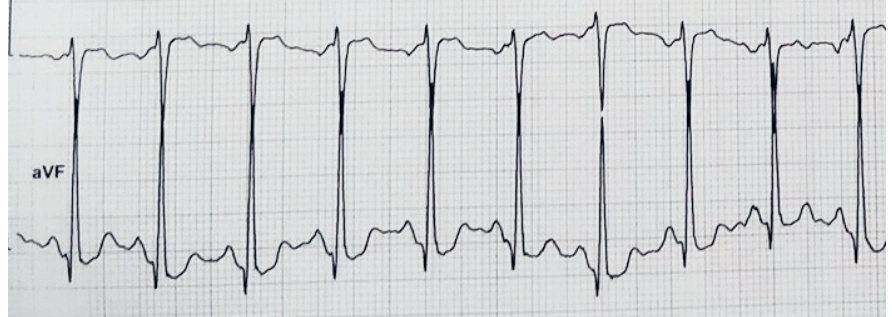

Figure 6. Slight ST depression in Inferior leads, recovery phase (masked group) 


\section{Discussion}

The current study provides valuable insights regarding use of surgical masks in exercise. While we have some limitations, the results still are relevant. The small number of subjects is important to consider, but the previous studies reported are also with small numbers (maximal 14 subjects) and the need to evaluate the research question rapidly in the context on a Pandemic Crisis made us prefer less subjects but a faster conclusion. There is a majority of males in our evaluation, but this can simply be a representation of the number of people exercising, not a bias Per Se. We chose young healthy volunteers since, assuming the possibility of harm with mask use while exercise, we did not considered ethical to test patients with cardiac pathology already diagnosed. Also, our volunteers did at least some physical activity, so the adaptation to the treadmill test was easier. Health status was reported by our subjects, but all were health professionals, so we accepted the claim. In our study subjects were not required to control their average physical activity, to abstain from exercise in specific periods or to control their eating habits before the study. This had the purpose of not interfering with the daily routines of our subjects and, since they were acting as their own control, obtain a result with higher external validity. Performing the tests in the same location with the same machine in a 2 week difference also contributed to the validity of the results, since this rules out interfering aspects like exercising in different weather patterns, at different times of the day or in different environments (roads, sports track, etc). The study by Fikenzer et al. (2020) did showed changes in respiratory parameters like forced expiratory volume and peak expiratory flow, but neither oxygen saturation nor physical activity performed was evaluated. Shaw et al (2020) however, did test oxygen saturation levels between rest and peak exercise and total physical activity performed. In this study neither of these parameters was statistically significant. In our study this was not the case. Both the physical load performed (estimated by our MET-Score) and oxygen saturation (O2Sat) show clear differences when subjects wore masks. Physical capacity and O2Sat at peak exercise were decreased when subjects wore a mask compared to not wearing one, showing that indeed the use of mask can cause measurable respiratory and exercise capacity changes even in healthy volunteers doing exercise. When we compare our data with the one from Shaw et al. (2020) it is possible that the fact that we used a treadmill test and not a cycle ergometer might have affected the results regarding O2Sat. Since higher muscle mass is used in a treadmill that in a cycle ergometer (Kisan, Kisan, Or, Sp, 2012) this could theoretically cause a higher oxygen demand. The notion is not without its merits, if we considerer that at least for patients with chronic obstructive pulmonary disease a treadmill test has been described as eliciting lower oxygen saturation levels at peak exercise vs a cycle ergometer (Hsia, Casaburi, Pradhan, Torres, Porszasz, 2009). It has been shown that the maximal oxygen consumption $\left(\mathrm{VO}_{2 \max }\right)$ is higher in treadmill stress tests compared with cycle ergometer stress tests in trained individuals (Basset, Boulay, 2000) which in theory would convey a higher aerobic capacity and therefore a possible higher exercise capacity in that time of activity. Although our methods are different, we clearly had a difference with the use of a mask. We preferred a treadmill because it's more commonly used in our country and we believe replicates more accurately the type of leisure exercise most healthy subjects do (jogging), so we believe our results can be more relevant to the general population, notwithstanding the small numbers used.

Eliciting the same results we had doing a different strategy, like performing a high intensity period of exercise done in a short amount of time, replicating protocols like those of High Intensity Interval Training (Tabata, Irisawa, Kouzaki, Nishimura, Ogita, Miyachi, 1997) could be an option to verify the changes we saw in a less time consuming matter. Further studies might be helpful in this matter. 
A quite surprising result obtained, although not reaching statistically significance, was the appearance of non diagnostic but doubtful ECG changes in subjects while doing the test with a mask that disappeared when the test was repeated without mask. Since all subjects were self-reported healthy, asymptomatic, and the unmasked exam was done two weeks later, without any medication or change in habits, we do not believe this was related to ischemia. While we could speculate that an unusually high change in O2Sat between rest and peak could be that cause of this, that did not happen in our subjects, since the changes were slight. Also, it is questionable that such hypoxic effect could cause such changes unless it's quite significant (Entwistle, Sommerville, Tandon, Jones, 1994; Coustet, Lhuissier, Vincent, Richalet, 2015) These subjects will perform further evaluations to rule-out the possibility of other alterations.

Since the beginning of this pandemic period, it has been regular practice to perform treadmill tests for diagnosis of coronary artery disease with the patients wearing masks. Considering our findings regarding the ECG changes and the exercise capacity (Roger et al., 1998) the possibility of diagnostic interference with mask use exists and has to be taken into account, and cardiologists should be aware of this while interpreting studies. Most subjects that do a treadmill stress tests do so to evaluate cardiovascular parameters in order to diagnose pathology. If the use of a mask can cause healthy volunteers to perform less exercise (and, as we saw, elicit ECG changes), than lower exercise levels performed might make a cardiologist believe a problem exists where he is simply detecting limitation caused by the use of a mask, and so the false positive results might increase.

Another relevant issue is, since we did detect significant changes in exercise capacity, oxygen saturation and reported discomfort with mask use, if this might impact the amount and level of exercise performed by the everyday subject if a mask is mandatory in these situations: While for the trained athlete the decrease in exercise load is the main issue (this might be relevant also for the casual subject that does leisure physical activity, since it might demoralize someone that's starting to exercise), the discomfort and the decrease in O2Sat might be much more significant for the casual person, and might cause an increased abandonment of exercise.

From our results, if viable from the epidemiological perspective, exercise should be done without a mask, and consideration for exercise stress tests without masks has to be considered.

\section{Conclusion}

Our study shows that young healthy subjects have significant decreases in exercise capacity and oxygen saturation when performing exercise using surgical masks, besides reporting discomfort with its use. Also, there were slight ECG changes only present with mask use, which could cause difficulties in diagnosis of ischemia. A study with a higher number of subjects might clarify these findings, but we believe exercise should be done without a mask and consideration for exercise stress tests without masks should be discussed in the Medical Community.

\section{Referenences}

Basset, F.A., Boulay, M.R. (2000). Specificity of treadmill and cycle ergometer tests in triathletes, runners and cyclists. Eur J Appl Physiol, 81 (3), 214-21. DOI: 10.1007/s004210050033.

Bruce, R.A., Blackmon, J.R., Jones, J.W., Strait, G. (1963). Exercise testing in adult normal subjects and cardiac patients. Pediatrics, 32, 742-756.

CDC (2020). Recommendation regarding the use of cloth face coverings, especially in areas of significant community-based transmission. 
Chandrasekaran, B., Fernandes, S. (2020). "Exercise with Facemask; Are We Handling a devil's sword?" - A Physiological Hypothesis. Med. Hypothesis, 144, 110002. DOI: 10.1016/j.mehy.2020.110002.

Coustet, B., Lhuissier, F.J., Vincent, R., Richalet, J.P. (2015). Electrocardiographic changes during exercise in acute hypoxia and susceptibility to severe high-altitude illnesses. Circulation, 131 (9), 786-794. DOI: 10.1161/CIRCULATIONAHA.114.013144.

ECDC (2020). Using face masks in the community - reducing COVID-19 transmission from potentially asymptomatic or presymptomatic people through the use of face masks.

Entwistle, M.D., Sommerville, D., Tandon, A.P., Jones, J.G. (1994). Effect of hypoxaemia on the resting electrocardiogram (ECG) in patients with cardiac ischaemia. Ann Acad Med Singap, 23 (4), 460-464.

Fikenzer, S., Uhe, T., Lavall, D. Rudolph, U., Falz, R., Busse, M., Hepp, P., Laufs, U. (2020). Effects of surgical and FFP2/N95 face masks on cardiopulmonary exercise capacity. Clin Res Cardiol, 109 (12): 1522-1530. DOI: 0.1007/s00392-020-01704-y.

Fletcher, G.F., Ades, P.A., Kligfield, P., Arena, R., Balady, G.J., Bittner, V.A., Coke, L.A., Fleg, J.L., Forman, D.E., Gerber, T.C., Gulati, M., Madan, K., Rhodes, J., Thompson, P.D., Williams, M.A. (2013). American Heart Association Exercise, Cardiac Rehabilitation, and Prevention Committee of the Council on Clinical Cardiology, Council on Nutrition, Physical Activity and Metabolism, Council on Cardiovascular and Stroke Nursing, and Council on Epidemiology and Prevention. Exercise standards for testing and training: a scientific statement from the American Heart Association. Circulation, 128 (8), 873-934. DOI: 10.1161/CIR.0b013e31829b5b44.

Garner, K.K, Pomeroy, W., Arnold, J.J. (2017). Exercise Stress Testing: Indications and Common Questions. Am Fam Physician, 96 (5), 293-299.

Gibbons, R.J., Balady, G.J., Beasley, J.W., Bricker, J.T., Duvernoy, W.F., Froelicher, V.F., Mark, D.B., Marwick, T.H., McCallister, B.D., Thompson, P.D., Winters, W.L. Jr., Yanowitz, F.G., Ritchie, J.L., Cheitlin, M.D., Eagle, K.A., Gardner, T.J., Garson, A.Jr, Lewis, R.P., O'Rourke, R.A., Ryan, T.J. (1997). ACC/AHA guidelines for exercise testing: executive summary. A report of the American College of Cardiology/American Heart Association Task Force on Practice Guidelines (Committee on Exercise Testing). Circulation, 96 (1), 345-54.

Greenhalgh, T. (2020). Face coverings for the public: Laying straw men to rest. J Eval Clin Pract., 26: 1070-1077. DOI: $10.1111 /$ jep.13415.

Hsia, D., Casaburi, R., Pradhan, A., Torres ,E., Porszasz, J. (2009). Physiological responses to linear treadmill and cycle ergometer exercise in COPD. Eur Respir J, 34 (3), 605-615.

Kisan, R., Kisan, S.R., Or, A., Sp, C. (2012). Treadmill and Bicycle Ergometer Exercise: Cardiovascular Response comparison. The Journal of medical research, $12(5)$.

Roger, V.L., Jacobsen, S.J., Pellikka, P.A., Miller, T.D., Bailey, K.R., Gersh, B.J. (1998). Prognostic value of treadmill exercise testing: a population-based study in Olmsted County, Minnesota. Circulation, 98 (25), 2836-2841. DOI: 10.1161/01.cir.98.25.2836.

Shaw, K., Butcher, S., Ko, J., Zello, G.A., Chilibeck, P.D. (2020). Wearing of Cloth or Disposable Surgical Face Masks has no Effect on Vigorous Exercise Performance in Healthy Individuals. Int J Environ Res Public Health, 17 (21), 8110. DOI: 10.3390/ ijerph17218110.

Tabata, I., Irisawa, K., Kouzaki, M., Nishimura, K., Ogita, F., Miyachi, M. (1997). Metabolic profile of high intensity intermittent exercises. Med Sci Sports Exerc, 29 (3), 390-395. DOI: 10.1097/00005768-199703000-00015.

Citte this article aS: Mendonça Café, H., Leitão, M., Freitas, A., Marreiros, A. (2021). Surgical Mask Use in Physical Exercise in Young Healthy Subjects Sume Trial. Central European Journal of Sport Sciences and Medicine, 3 (35), 117-125. DOI: 10.18276/ cej.2021.3-10. 